\title{
Molecular characterization of H3N2 and H4N6 subtypes avian influenza viruses isolated from mallards in Poyang Lake, China in 2010
}

\author{
ZHU Na ${ }^{1}$, ZHAO JiuRu $^{1}$, LI YongDong ${ }^{2}$, DING ChangQing ${ }^{3}$, XIA Han ${ }^{1}$, TANG Shuang ${ }^{1}$, \\ ZHANG Zhong ${ }^{1}$, YU JingFeng ${ }^{1}$, CHEN Jing ${ }^{1}$, FAN ZhaoJun ${ }^{1}$, KOU Zheng ${ }^{1 *}$ \& LI TianXian ${ }^{1 *}$ \\ ${ }^{1}$ State Key Laboratory of Virology, Wuhan Institute of Virology, Chinese Academy of Sciences, Wuhan 430071, China; \\ ${ }^{2}$ Ningbo Municipal Center for Disease Control and Prevention, Ningbo 315010, China; \\ ${ }^{3}$ College of Biology, Beijing Forestry University, Beijing 100083, China
}

Received February 10, 2012; accepted May 14, 2012; published online July 10, 2012

\begin{abstract}
Poyang Lake is the largest inland freshwater lake in China and contains many species of wild birds and waterfowls. We conducted a survey of avian influenza viruses in nine semi-artificial waterfowl farms in Poyang Lake during January to March of 2010. Out of 1036 cloacal swabs collected, three H3N2 and one H4N6 influenza viruses were isolated from healthy mallards. All the isolates were genetically and phylogenetically characterized. The analysis of putative $H A$ cleavage sites showed that all the four isolates possessed the molecular characteristics (QTRGL for H3N2 viruses, PEKASR for H4N6 virus) of lowly pathogenic avian influenza (LPAI) virus. The phylogenetic analysis of the viral genomes showed that all four virus isolates clustered in the Eurasian clade of influenza viruses. The $M$ gene of the viruses possessed the highest homology with highly pathogenic H5N1 influenza viruses. In addition, co-infection of H3N2 and H4N6 in the same farm was observed. And interestingly, we isolated two subtypes viruses (H3N2 and H4N6) and their progeny virus (H3N2) with evidence of genome reassortment from the same farm, in which the $P B 1$ and $P B 2$ gene segments of H4N6 replaced those of the H3N2 strain. The results of animal infection experiments showed that all the four isolated viruses were lowly pathogenic to chickens and not pathogenic to mice, which was consistent with the results of genetic analysis.
\end{abstract}

influenza A virus, Poyang Lake, epidemic survey, evolution

Citation: Zhu N, Zhao J R, Li Y D, et al. Molecular characterization of H3N2 and H4N6 subtypes avian influenza viruses isolated from mallards in Poyang Lake, China in 2010. Chin Sci Bull, 2012, 57: 3586-3594, doi: 10.1007/s11434-012-5312-y

Influenza A virus (IAV), a member of the Orthomyxoviridae family of viruses, can be further categorized into different subtypes based on the antigenicity of the surface glycoproteins, the hemagglutinin $(H A)$ and neuraminidase (NA). Until now $16 \mathrm{HA}$ subtypes and nine $N A$ subtypes have been identified [1]. IAVs have a wide range of host species, including humans, pigs, horses, marine mammals, and a variety of birds [2,3]. All IAV subtypes have been isolated from waterfowls $[1,4,5]$, with waterfowls as their main natural hosts.

\footnotetext{
*Corresponding authors (email: litx@wh.iov.cn (T. Li); kouzheng@wh.iov.cn (Z. Kou))
}

Different subtypes of avian influenza virus have spread worldwide, especially the rapid spread of the highly pathogenic $\mathrm{H} 5 \mathrm{~N} 1$ avian influenza that poses a high risk to the health of humans and birds. In 2005, the outbreak of highly pathogenic H5N1 avian influenza at Qinghai Lake caused the death of more than 6000 wild migratory birds [2,6], followed by outbreaks of avian flu in Europe, other Asian countries, and some African countries. In recent years, avian flu outbreaks in wild birds and domestic fowls have occurred frequently in mainland China [7-11].

Poyang Lake is the largest inland freshwater lake in China. Located in Central China, it is an important migra- 
tory bird stopover point and wintering ground, during which times, a large number of wild birds (including 108 species of waterfowl) can be found at the lake. The poultry industry for both domestic fowl and waterfowl are highly developed around Poyang Lake. The semi-artificial mode of poultry production is widely adopted by the local farmers, and a large number of wild bird species are farmed. The frequent contact between the wild birds and the domestic fowl has facilitated the spread of avian influenza viruses [12-15]. Therefore, enhanced monitoring of the wild birds and domestic fowls has become extremely important. In this study, we carried out an epidemiological investigation in nine waterfowl farms around Poyang Lake, in an attempt to provide the basis for the implementation of more effective prevention and control of avian influenza virus.

\section{Materials and methods}

\subsection{Sample collection}

During January to March of 2010, 1036 cloacal swab samples were collected from three species of waterfowls (greylag goose (Anser anser, $n=316$ ), mallard (Anas platyrhynchos, $n=422$ ), spot-billed duck (Anas poecilorhyncha, $n=$ 298)) in nine semi-artificial waterfowl farms located around Poyang Lake. All samples were immediately placed 2.0 $\mathrm{mL}$ plastic screw-capped tubes containing transfer solution, and then stored in liquid nitrogen while being transported to the laboratory. The transport medium consisted of phosphate-buffered saline (PBS)/Glycerol $(50 \%)$, containing antibiotics $\left(2 \times 10^{6} \mathrm{U} / \mathrm{L}\right.$ penicillin $\mathrm{G}$, $2 \times 10^{6} \mathrm{U} / \mathrm{L}$ polymyxin $\mathrm{B}, 0.5 \times 10^{6} \mathrm{U} / \mathrm{L}$ nystatin, $200 \mathrm{mg} / \mathrm{L}$ streptomycin, $250 \mathrm{mg} / \mathrm{L}$ gentamicin, $60 \mathrm{mg} / \mathrm{L}$ Ofloxacin $\mathrm{HCl}$, and $200 \mathrm{mg} / \mathrm{L}$ sulfamethoxazole) $[16,17]$.

\subsection{Identification of IAV}

(i) RNA extraction and cDNA synthesis. For cloacal swabs, RNA was extracted using Trizol reagent (Invitrogen, Carlsbad, CA, USA), according to the manufacturer. Transport medium $(250 \mu \mathrm{L})$ was used to extract RNA, and then RNA was eluted using $30 \mu \mathrm{L}$ diethylpyrocarbonate (DEPC)-treated $\mathrm{H}_{2} \mathrm{O}$. Next, $6 \mu \mathrm{L}$ of the eluate containing RNA was reverse transcribed into cDNA using AMV reverse transcriptase (Promega, Madison, WI, USA) and 500 ng Unit12 primer in $40 \mu \mathrm{L}$ reaction system according to the protocol, the primer for reverse transcription of viral RNA genome was 5'-AGCAAAAGCAGC-3' [18]. The reverse transcription reaction was performed at $37^{\circ} \mathrm{C}$ for $55 \mathrm{~min}$, and cDNA was stored at $-20^{\circ} \mathrm{C}$.

(ii) Real-time quantitative PCR. IAV was detected using a real-time quantitative polymerase chain reaction (PCR) assay targeting the matrix $(M)$ gene, as described in a previous report [19]: 5'-CTTCTAACCGAGGTCGAAACG-3' for forward primer, 5'-AGGGCATTTTGGACAAAGCGTCTA-3' for reverse primer, 5'-FAM-TTTGTGTTCACGCTCACCGTGCC-TAMRA-3' for probe. In brief, each reaction mixture included $2.5 \mu \mathrm{L}$ of $10 \times$ Ex Taq Buffer, 3 $\mu \mathrm{L} 2.5 \mathrm{mmol} / \mathrm{L} \mathrm{dNTP}, 3.5 \mu \mathrm{L} 25 \mathrm{mmol} / \mathrm{L} \mathrm{MgCl}_{2}, 0.5 \mu \mathrm{L}$ of each primer $(20 \mu \mathrm{mol} / \mathrm{L}), 1 \mu \mathrm{L} 10 \mu \mathrm{mol} / \mathrm{L}$ TaqMan probe, $0.25 \mu \mathrm{L}$ Ex Taq, $1 \mu \mathrm{L}$ BSA (0.05\%), $1 \mu \mathrm{L}$ cDNA and add DEPC $\mathrm{H}_{2} \mathrm{O}$ to $25 \mu \mathrm{L}$. Cycling conditions in Lightcycler 2.0 (Roche, Mannheim, Germany) were as follows: $95^{\circ} \mathrm{C}$ for 10 min, and 45 cycles of $95^{\circ} \mathrm{C}$ for $20 \mathrm{~s}$ and $60^{\circ} \mathrm{C}$ for $1 \mathrm{~min}$. When samples gained the threshold cycle $(\mathrm{Ct})$ value, they were regarded as $M$ gene positive in this study. A positive control and negative control were also placed in parallel in the real-time PCR. And the resulting Ct value of the positive control was approximately 30 .

\subsection{Virus isolation}

Real-time quantitative PCR positive samples were subsequently processed for virus isolation. Virus isolation from the samples was carried out using 10-d-old specificpathogen-free (SPF) embryonated chicken eggs (Merial, Beijing, China). Prior to inoculation into eggs, the samples in PBS were centrifuged at $3000 \times g$ for $5 \mathrm{~min}$ at $4^{\circ} \mathrm{C}$. Supernatants were supplemented with penicillin G (40000 $\mathrm{IU} / \mathrm{mL})$, streptomycin sulfate $(8000 \mathrm{IU} / \mathrm{mL})$ and were kept overnight at $4^{\circ} \mathrm{C}$. The sample supernatant was inoculated into the allantoic cavity of each egg (2-3 eggs per sample). After incubation at $37^{\circ} \mathrm{C}$ for $24-72 \mathrm{~h}$, the allantoic fluid was harvested and IAV was detected using hemagglutination assays with rooster erythrocytes. All allantoic fluids containing virus stocks were stored at $-70^{\circ} \mathrm{C}$.

\subsection{Nucleotide sequencing and phylogenetic analysis}

As previously described, total RNA extracted from allantoic fluids was transcribed into cDNA using the Unit12 primer (5'-AGCRAAAGCAGG-3') and the cDNA samples were used as template for PCR to amplify the full length of all genes using the primer sets [18]. The PCR products were routinely processed with $1 \%$ agarose gel electrophoresis and pieces of gel containing DNA bands of expected sizes were cut out and purified using a gel extraction kit (Axygen, Union City, CA, USA). The purified PCR products were sequenced using an ABI PRISM 370 DNA sequencer (Applied Biosystems, Carlsbad, CA, USA). All sequences have been deposited in GenBank under accession numbers: CY098243 - CY098274. For phylogenetic analysis, nucleotide sequences of H3N2 and H4N6 representing different geographic locations, host species and Eurasian/North American lineages were included. All sequence data were edited using BioEdit (version 5.0) and phylogenetic trees were generated using the neighbor-joining (NJ) method supported by 1000 bootstrap replicates in MEGA (version 4.0). 


\subsection{Animal experiment}

To determine the pathogenicity of the four isolates, four groups of ten SPF White Leghorn chickens (Merial) aged six weeks were intravenously inoculated with $0.2 \mathrm{~mL}$ of a 1:10 dilution of allantoic fluid that contained virus $\left(10^{6}\right.$ $\left.\mathrm{EID}_{50}\right)$ and observed for clinical signs of disease and mortality for $14 \mathrm{~d}$ [20]. On the 3rd day of post infection (p.i.), three chickens of each group were sacrificed and tissue samples from lung were collected for detection of virus by real-time quantitative PCR as previously described. Control groups of chickens were inoculated with PBS.

In order to evaluate the isolates replication ability in BALB/c mice (Experiment Animal Center of Hubei Control Disease Center, Wuhan, China), four groups of ten 6-weekold female BALB/c mice were infected intranasally with each virus in a volume $50 \mu \mathrm{L}$ of $10^{6} \mathrm{EID}_{50}$, under anesthesia [21]. On day 3 , three mice of each group were sacrificed and the organs were collected for detection of virus by real-time quantitative PCR as previously described. The remaining inoculated mice were monitored daily for mortality and clinical signs of disease. Control groups of mice were inoculated with PBS.

\section{Results}

\subsection{Virus isolation}

Among the 1036 samples, 49 samples were $M$ gene positive by real-time quantitative PCR. And four avian influenza viruses were isolated from the 49 positive cloacal swabs samples based on embryonated chicken eggs (Table 1). The whole genomes of all the isolated viruses were sequenced to analysis the genetic character of the viruses.

Among the four isolates, the $H A$ and $N A$ genes of three isolates showed high similarity with each other $(99.0 \%-$ 99.5\%) and had highest similarity (94\%-95\%) with those of A/white-backed munia/Hong Kong/4519/2009(H3N2), so these three viruses were shown to belong to subtype H3N2 and were designated as A/Mallard/Jiangxi/1-4/2010(H3N2) (ML/JX/1-4/10), A/Mallard/Jiangxi/1-19/2010(H3N2) (ML/ JX/1-19/10), A/Mallard/Jiangxi/2-5/2010(H3N2) (ML/JX/ 2-5/10). The $H A$ and $N A$ genes of the other one virus had the highest similarity (98\%-99\%) with A/avian/Japan/ 8KI0185/2008(H4N6), therefore, the isolate was shown to belong to subtype $\mathrm{H} 4 \mathrm{~N} 6$ and was designated as $\mathrm{A} /$ Mallard/ Jiangxi/1-15/2010(H4N6) (ML/JX/1-15/10).

Besides, among the four isolates, ML/JX/1-4/10(H3N2), $\mathrm{ML} / \mathrm{JX} / 1-15 / 10(\mathrm{H} 4 \mathrm{~N} 6)$, and ML/JX/1-19/10(H3N2) isolated from the same farm, and ML/JX/2-5/10(H3N2) was from another one farm.

\subsection{Genetic analysis}

All the H3N2 viruses shared the same amino acid sequence (QTRGL) at the HA1-HA2 cleavage site, and the H4N6 virus possessed PEKASR at the cleavage site. The cleavage site sequences of all viruses were characteristic of LPAI viruses. In all the four viruses, the receptor binding pocket of the HA1 retain amino acid residues 226-Gln and 228-Gly that preferentially bind to $\alpha-2,3-\mathrm{NeuAcGal}$ linkages of avian cell-surface receptors [22] and other amino acid residues relevant to receptor binding were identical to those of most avian influenza viruses of the same subtype. All viruses had His at position 274 of the neuraminidase protein, which were predicted to be sensitive to oseltamivir. All viruses did not have mutations of amino acids (Leu26, Val27, Ala30, Ser31) in the transmembrane region of the M2 protein, which had been shown to be associated with amantadine resistance [23]. In NS1, the four isolates do not contain the five amino acid deletions and D92 at position 92 has remained unaltered, which are both related to virulence in mammalian species [24]. All four isolates contained ESEV residues at the C-terminus of NS1 and none contained the E627K mutation in $P B 2$ gene.

\subsection{Phylogenetic analysis}

Phylogenetic analysis indicated that all the eight gene segments of the four viruses clustered in the Eurasian lineage. As shown in Figure 1, the $H A$ and $N A$ genes of H3N2 strains from Poyang Lake were grouped into the Eurasian lineage, and three $\mathrm{H} 3 \mathrm{~N} 2$ isolates had a relatively close genetic relationship with each other, clustered closely with those of A/white-backed munia/Hong Kong/4519/2009 (H3N2). As for the H4N6 strain, the results from phylogenetic analysis indicated that the $H A$ and $N A$ genes belonged to the lineage of avian viruses isolated in the Eurasian region, clustered closely with those of A/avian/Japan/ 8KI0185/2008(H4N6) (Figure 2). The phylogenetic analysis

Table 1 Samples from wild birds in the study

\begin{tabular}{lcccc}
\hline \multicolumn{1}{c}{ Species } & No. sampled $(n)$ & No. PCR positive $(n)$ & No. AI isolates $(n)$ & Prevalence $(\%)$ \\
\hline Anser anser & 316 & 6 & 0 & 0 \\
Anas poecilorhyncha & 298 & 4 & 0 & 0 \\
Anas platyrhynchos & 422 & 39 & 4 & 0.9 \\
Total & 1036 & 49 & 4 & 0.4 \\
\hline
\end{tabular}


(a)

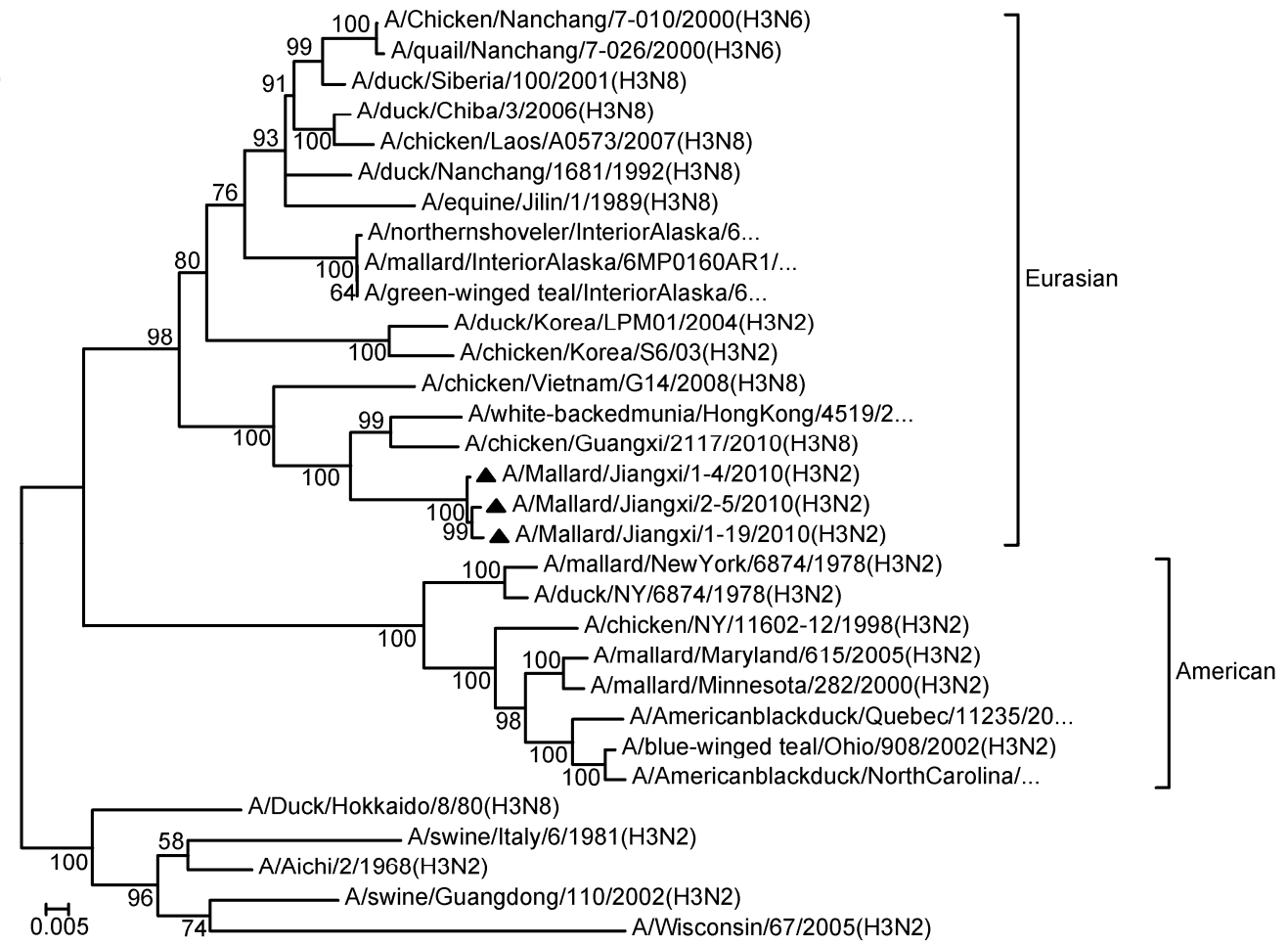

(b)

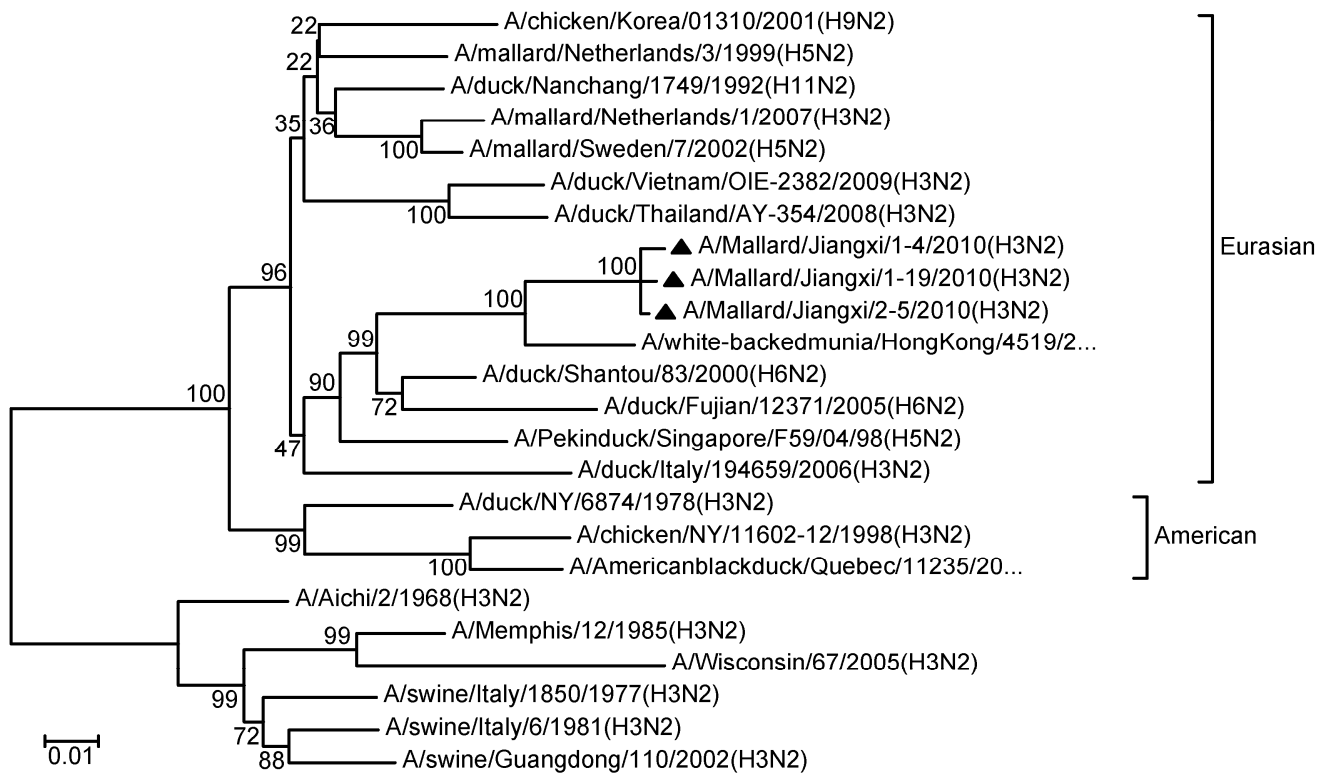

Figure 1 Phylogenetic trees of $H A$ and NA genes of subtype H3N2 strains isolated from Jiangxi Province, China. Trees were generated by using neighborjoinning analysis in MEGA (version 4.0). Numbers below branches indicate bootstrap value percent from 1000 replicates. (a) H3 (nucleotide 30 to 1717 bp); (b) N2 (nucleotide 5 to $1414 \mathrm{bp}$ ). The scale bar shows the mutation rate between each two sequences. The genes studied in this paper are represented by the solid triangles.

results were consistent with those of similarity analysis.

Phylogenetically, all the internal genes were divided into American and Eurasian lineages, the Eurasian lineage formed two groups: I and II, and all the six internal genes of the four viruses belonged to the Eurasian lineage. Phylogenetic analysis of the $P A, N P$ and $N S$ genes showed that ML/JX/1-15/10(H4N6) formed an independent branch ingroup I, whereas ML/JX/1-4/10(H3N2), ML/JX/1-19/10
(H3N2), and $\mathrm{ML} / \mathrm{JX} / 2-5 / 10(\mathrm{H} 3 \mathrm{~N} 2)$ clustered together in group II (Figure 3).

Phylogenetic analysis of the $M$ gene showed that all the four viral isolates were in the Group I clade, among which ML/JX/1-4/10(H3N2), ML/JX/1-19/10(H3N2), and ML/JX/ $2-5 / 10(\mathrm{H} 3 \mathrm{~N} 2)$ had a close genetic relationship, whereas their genetic relationship with ML/JX/1-15/10(H4N6) was relatively distant. It was noteworthy that the $M$ genes of 

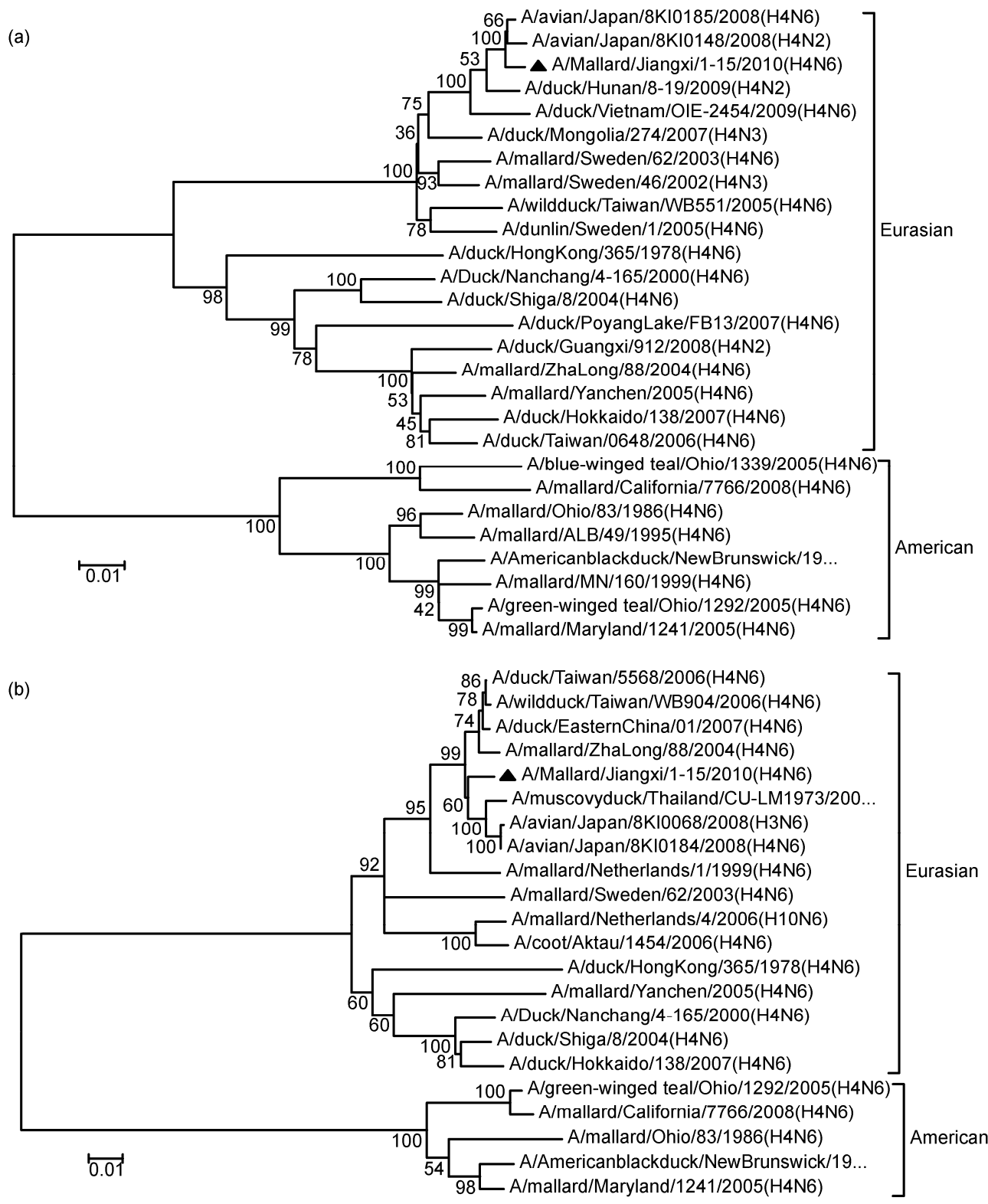

Figure 2 Phylogenetic trees of $H A$ and NA genes of subtype H4N6 strains isolated from Jiangxi Province, China. Trees were generated by using neighbor-joinning analysis in MEGA (version 4.0). Numbers below branches indicate bootstrap value percent from 1000 replicates. (a) H4 (nucleotide 20 to 1709 bp); (b) N6 (nucleotide 19 to 1423 bp). The scale bar shows the mutation rate between each two sequences. The genes studied in this paper are represented by the solid triangles.

four viral isolates shared the highest level of similarity with the $M$ genes from highly pathogenic H5N1 influenza viruses. ML/JX/1-4/10(H3N2), ML/JX/1-19/10(H3N2), and ML/JX/ $2-5 / 10(\mathrm{H} 3 \mathrm{~N} 2)$ shared the highest similarity $(99 \%)$ with A/mute swan/Aktau/1460/2006(H5N1); ML/JX/1-15/10 (H4N6) shared the highest similarity (95\%) with A/chicken/ Jilin/hk/2004(H5N1) (Figure 4).

In the phylogenetic trees of $P B 2$ and $P B 1$ genes, it showed that $\mathrm{ML} / \mathrm{JX} / 1-4 / 10(\mathrm{H} 3 \mathrm{~N} 2)$ and $\mathrm{ML} / \mathrm{JX} / 1-15 / 10$ (H4N6) formed a group, whereas ML/JX/1-19/10(H3N2) and $\mathrm{ML} / \mathrm{JX} / 2-5 / 10(\mathrm{H} 3 \mathrm{~N} 2)$ formed a separate cluster (Figure 5). It was clear that genome reassortment occurred in ML/JX/1-4/10(H3N2), such as the $P B 2$ and $P B 1$ genes of
H4N6 subtype replaced the corresponding gene segments of H3N2.

\subsection{Animal experiment}

To determine the pathogenicity of the isolates, four groups of chickens were inoculated and observed for clinical signs of disease and mortality for $14 \mathrm{~d}$. Three inoculated chickens of each group were sacrificed and tissue samples from lung were collected on day 3 after inoculation for virus detection. None of the chickens showed any clinical signs of disease within $14 \mathrm{~d}$ and none died during the observed period. The virus detection by the real-time quantitative PCR indicated 
(a)

61 A/whooperswan/Mongolia/6/2009(H5N1)

67 A/duck/Fukushima/2/2011(H5N1)

99 - A/greatcrestedgrebe/Tyva/22/2010(H5N1)

100 - A/bar-headedgoose/Mongolia/X53/2009(...

$100[$ A/chicken/Hunan/8/2008(H5N1)

- A/environment/DongtingLake/Hunan/3-9..

A A/Mallard/Jiangxi/1-15/2010(H4N6)

63 - A/duck/Korea/A93/2008(H5N2)

A/mallard/Netherlands/28/2006(H3N1)

A/mallard/Netherlands/26/2005(H11N2)

A/mallard/Italy/3401/2005(H5N1)
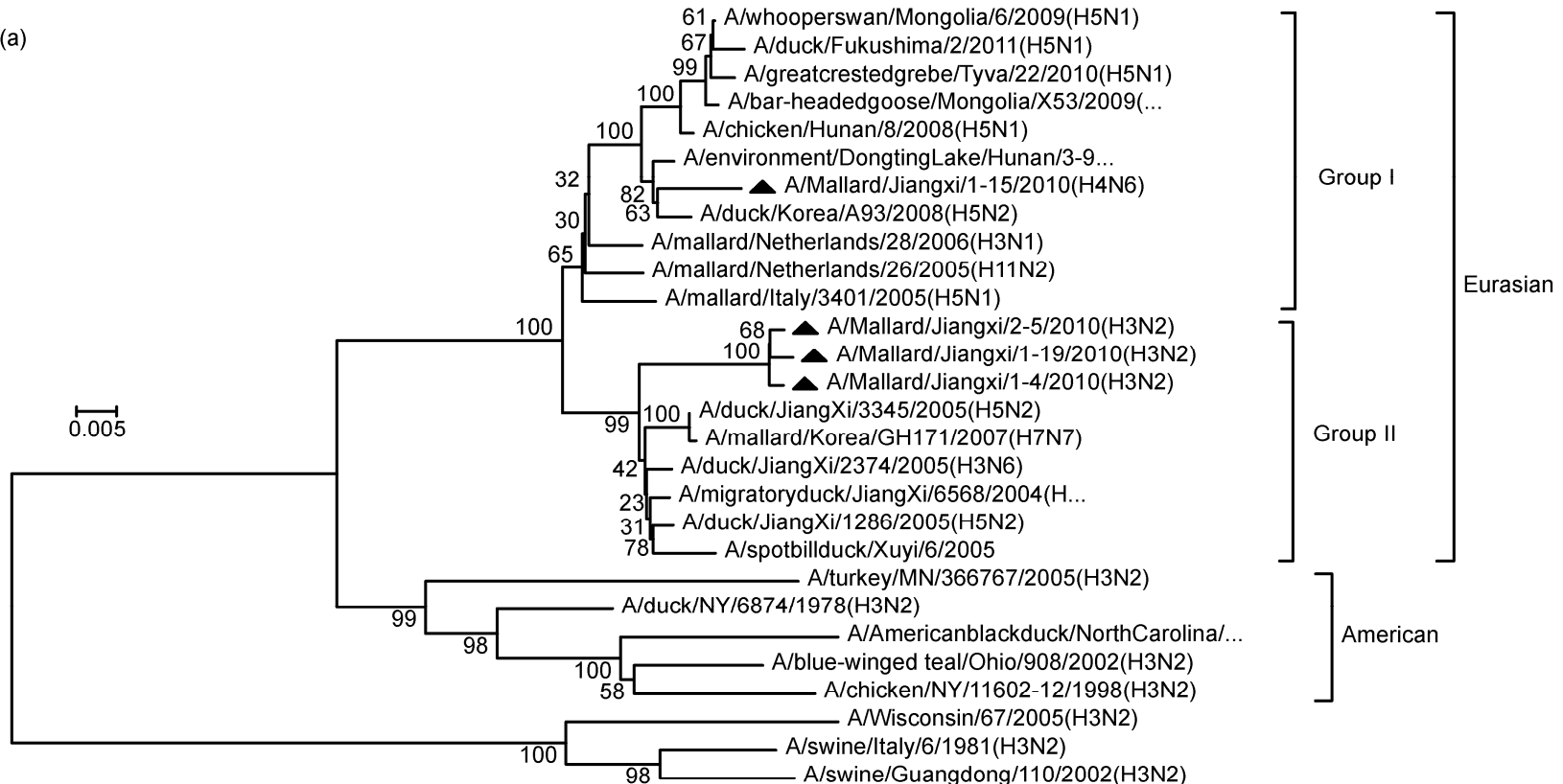

$98 \longrightarrow$ A/swine/Guangdong/110/2002(H3N2)
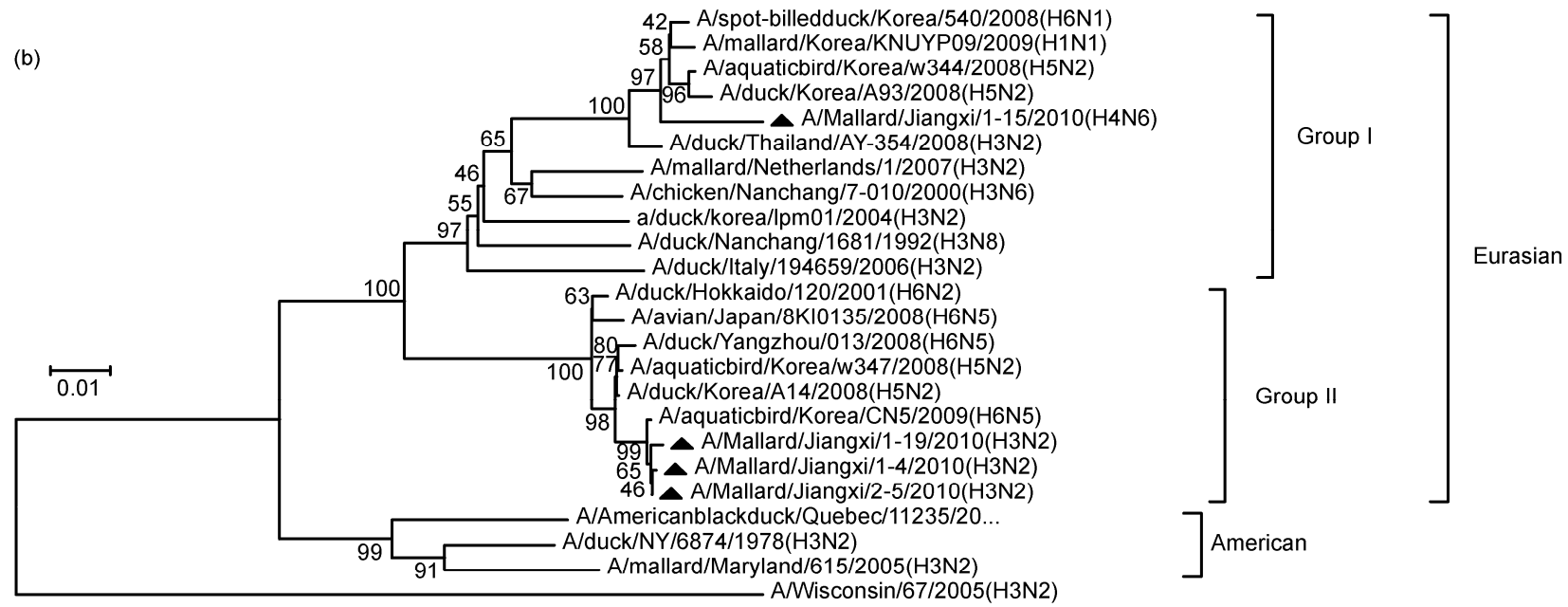

(c)

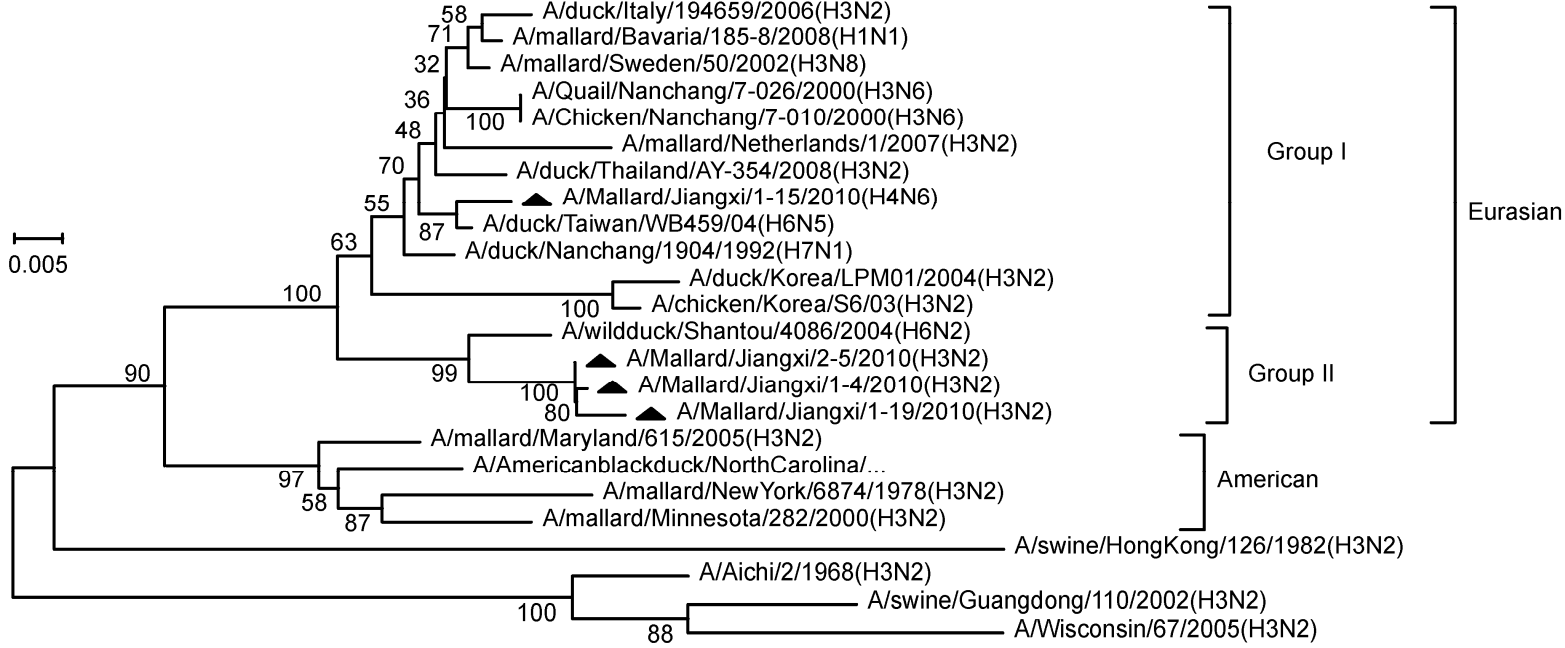

Figure 3 Phylogenetic trees of $P A, N P$ and $N S$ genes of strains isolated from Jiangxi Province, China. Trees were generated by using neighbor-joinning analysis in MEGA (version 4.0). Numbers below branches indicate bootstrap value percent from 1000 replicates. (a) $P A$ (nucleotide 8 to 2194 bp); (b) NP (nucleotide 31 to $1471 \mathrm{bp}$ ); (c) $N S$ (nucleotide 8 to $851 \mathrm{bp}$ ). The scale bar shows the mutation rate between each two sequences. The genes studied in this paper are represented by the solid triangles. 


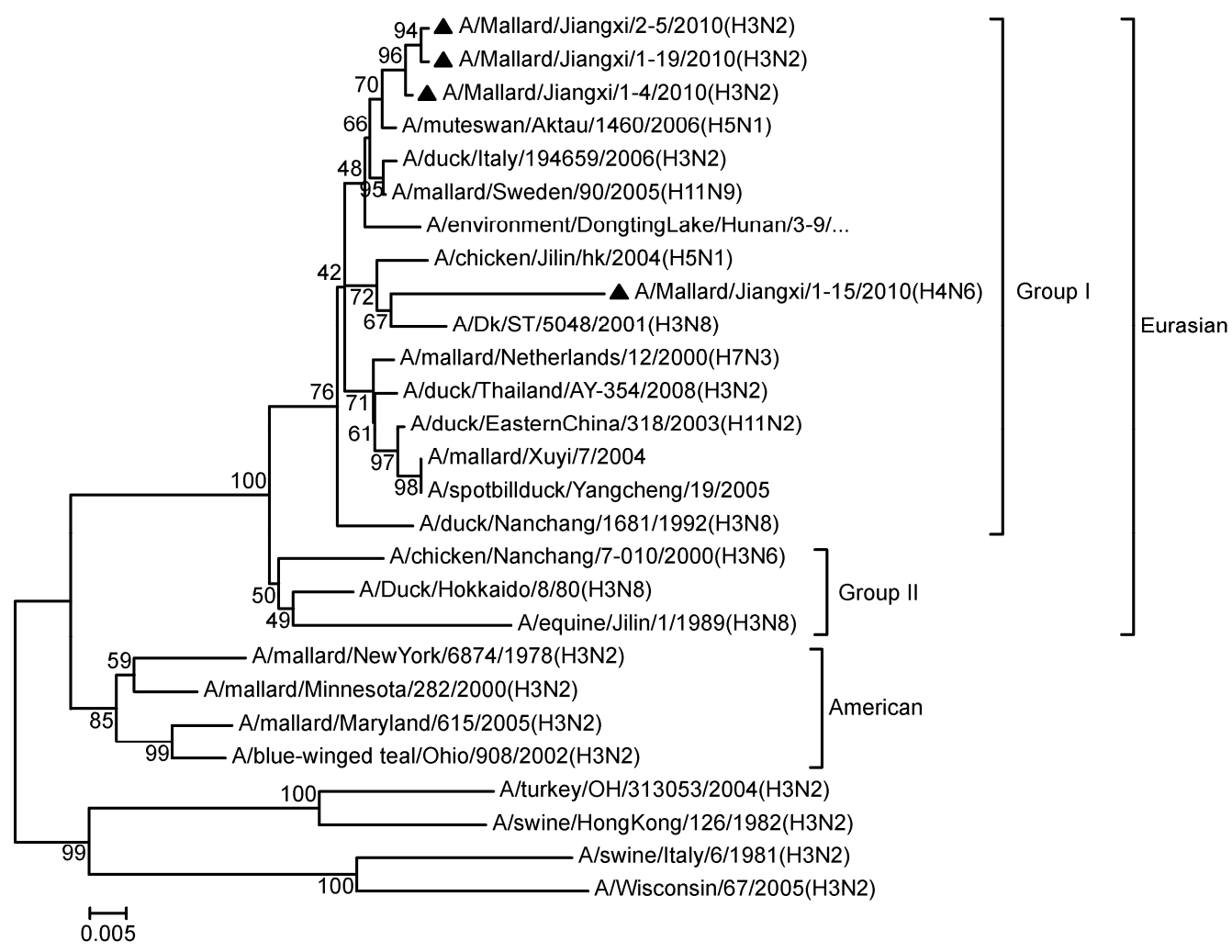

Figure 4 Phylogenetic trees of $M$ gene of strains isolated from Jiangxi Province, China. Trees were generated by using neighbor-joinning analysis in MEGA (version 4.0). Numbers below branches indicate bootstrap value percent from 1000 replicates. Analysis was based on nucleotide 31 to $1012 \mathrm{bp}$. The scale bar shows the mutation rate between each two sequences. The genes studied in this paper are represented by the solid triangles.

viral shedding in all lung samples on day 3 p.i. (Table 2). These results suggested that the isolates were lowly pathogenic to chickens, which was consistent with the results of genetic analysis.

We use BALB/c mice as a model to evaluate the ability of replication in the mammalian hosts of the isolated viruses. Four groups of ten 6 -week-old female BALB/c mice were infected intranasally with the virus in a volume $50 \mu \mathrm{L}$ of $10^{6}$ EID $_{50}$, under anesthesia. Three of ten inoculated mice were sacrificed on days 3 and 5 p.i. and their lungs, brain, spleen, and kidneys were collected for virus detection. No viral shedding was detected in any of the organs of the inoculated mice on days 3 or 5 after inoculation, and all the inoculated mice stayed healthy and kept gaining weight during the observation period. These results suggested that all the isolates were not pathogenic to mice.

\section{Discussion}

Poyang Lake is the largest inland freshwater lake in China and is located in the middle and lower reaches of the Yangtze River. This lake is rich in biodiversity and is the largest wintering ground for migratory birds in Asia. The special ecological environment of Poyang Lake has brought favorable conditions for the migration of wild birds, but also provides opportunities for the spread and genetic recombina- tion of influenza viruses. Given the special nature of local ecological environment and the genetic diversity of influenza virus, it is particularly important to monitor the possible spread of influenza viruses among wild birds and domestic fowls.

During January to March of 2010, 1036 cloacal swab samples were collected from three species of wild waterfowls in nine semi-artificial waterfowl farms around Poyang Lake. Forty-nine positive samples were detected by realtime quantitative PCR, and four influenza virus strains [ML/JX/1-4/10(H3N2), ML/JX/1-19/10(H3N2), ML/JX/2$5 / 10$ (H3N2), and ML/JX/1-15/10(H4N6)] were isolated. The four viral isolates identified in the present study were detected from two different farms around Poyang Lake. In fact, ML/JX/1-4/10(H3N2), ML/JX/1-15/10(H4N6), and ML/JX/1-19/10(H3N2) came from the same farm, suggesting the existence of the co-infection of different subtypes (H4N6 and H3N2). The genetic analysis showed that all the four isolated viruses possessed the molecular characteristics of LPAI viruses. And the results of animal infection experiments showed that all the four isolated viruses were lowly pathogenic to chickens and not pathogenic to mice, which was consistent with the results of genetic analysis.

Phylogenetic analysis indicated that all the eight gene segments of the four viruses clustered in the Eurasian lineage. Besides the phylogenetic analysis results showed that the $H A, N A, N S, M, N P$, and $P A$ gene segments of ML/JX/1- 

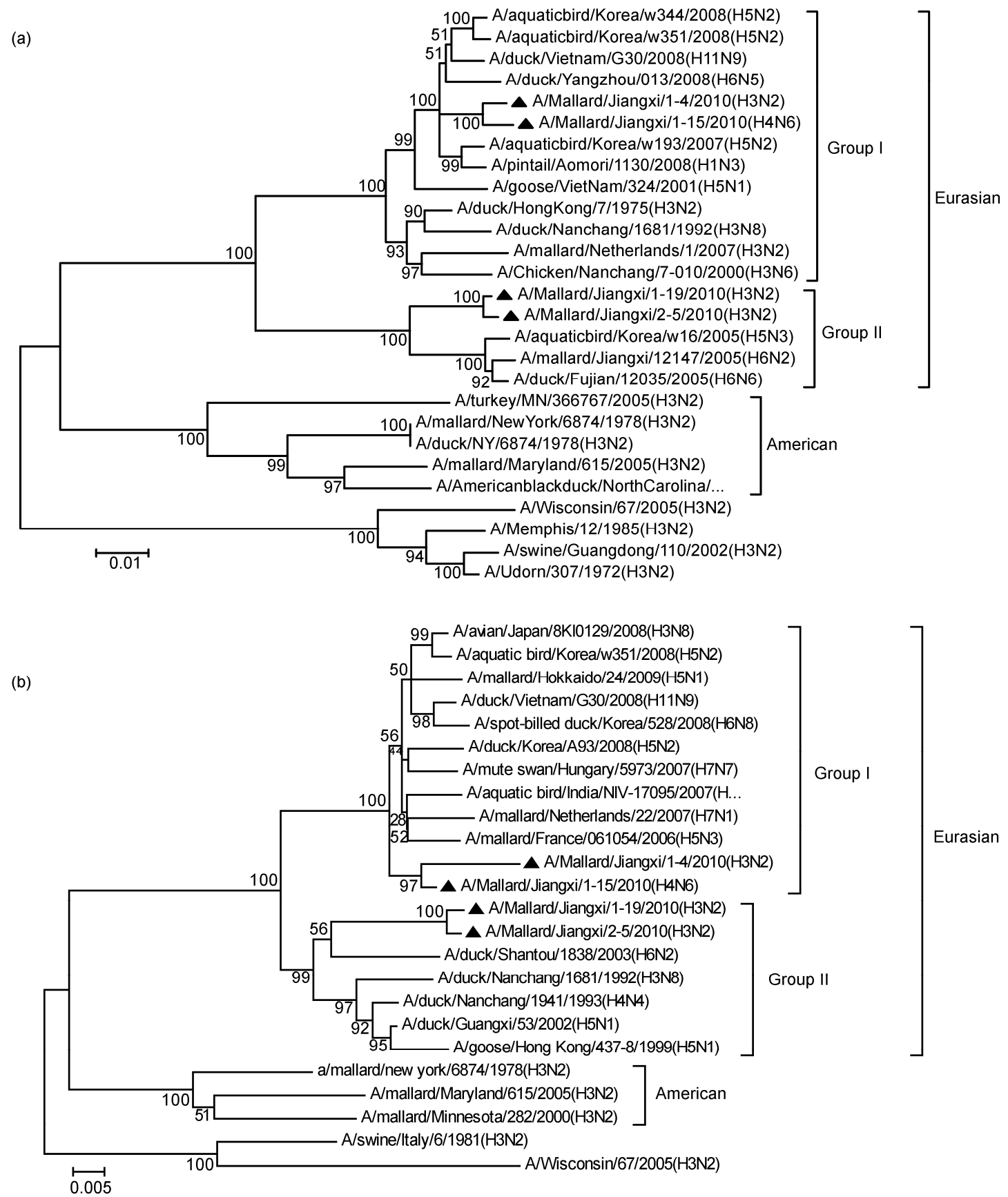

Figure 5 Phylogenetic trees of $P B 2$ and $P B 1$ genes of strains isolated from Jiangxi Province, China. Trees were generated by using neighbor-joinning analysis in MEGA (version 4.0). Numbers below branches indicate bootstrap value percent from 1000 replicates. (a) $P B 2$ (nucleotide 35 to 2242 bp); (b) PB1 (nucleotide 30 to $2303 \mathrm{bp}$ ). The scale bar shows the mutation rate between each two sequences. The genes studied in this paper are represented by the solid triangles.

Table 2 Replication of the viruses in chickens

\begin{tabular}{lccc}
\hline Viruses name & $\begin{array}{c}\text { No. of chickens shedding viruses/ } \\
\text { No. of chickens sacrificed }\end{array}$ & $\begin{array}{c}\text { Virus copies in lung } \\
\text { (mean } \log _{10} \text { copies/ } \mu \mathrm{L} \pm \mathrm{SD} \text { ) }\end{array}$ & $\begin{array}{c}\text { No. of death/ } \\
\text { No. of inoculation }\end{array}$ \\
\hline ML/JX/1-4/10 & $3 / 3$ & $5.93 \pm 0.006$ & $0 / 10$ \\
ML/JX/1-15/10 & $3 / 3$ & $5.64 \pm 0.06$ & $0 / 10$ \\
ML/JX/1-19/10 & $3 / 3$ & $4.98 \pm 0.07$ & $0 / 10$ \\
ML/JX/2-5/10 & $3 / 3$ & $5.44 \pm 0.03$ & $0 / 10$ \\
Control group & $0 / 3$ & 0 & $0 / 10$ \\
\hline
\end{tabular}


$4 / 10(\mathrm{H} 3 \mathrm{~N} 2)$ originated from $\mathrm{ML} / \mathrm{JX} / 1-19 / 10(\mathrm{H} 3 \mathrm{~N} 2)$ or $\mathrm{ML} / \mathrm{JX} / 2-5 / 10(\mathrm{H} 3 \mathrm{~N} 2)$. However, the gene segments of $P B 1$ and $P B 2$ originated from ML/JX/1-15/10(H4N6), suggesting that $\mathrm{ML} / \mathrm{JX} / 1-4 / 10(\mathrm{H} 3 \mathrm{~N} 2)$ was produced after genomic reassortment of influenza viruses of two subtypes, in which the $P B 1$ and $P B 2$ gene segments of H4N6 replaced those of the H3N2 strain. Furthermore, the phylogenetic analysis of $M$ gene showed that these four isolates were genetically close to two highly pathogenic H5N1 strains (A/chicken/ $\mathrm{Jilin} / \mathrm{hk} / 2004(\mathrm{H} 5 \mathrm{~N} 1)$ and A/mute swan/Aktau/1460/2006 (H5N1)), indicating the $M$ gene segment might be derived from highly pathogenic H5N1 viruses by reassortment. Genomic reassortants may be closely related with the local mode of feeding (scattered-feeding). Since the domestic fowls and wild birds are living in the same waters, genetic recombination among influenza viruses is likely.

All the samples in this study were obtained from semi-artificial wild waterfowl. The high density industrial poultry areas around Poyang Lake, especially the loose backyard poultry, increase the contacts among domestic poultry and wild birds, which may contribute to genome reassortment. Therefore, enhanced surveillance of the wild birds and domestic fowls, especially in communities with close association with humans, will be important to act as surveillance for public health.

This work was supported by the Ministry of Science and Technology of China (2010CB534005, 2007FY210700, 2009ZX10004-109), the National Natural Science Foundation of China (30970024, 30900060), Hubei Science and Technology Major Projects (2011ZX09401-302), the National $R \& D$ Infrastructure and Facility Development Program of China (BSDN2009-10\&18) and the Chinese Academy of Sciences (KSCX2YW-N-065, KSCX2-YW-R-157, 158 and 159, INFO-115-CO1-SDB3-01, INFO-115-C01-SDB4-21, IN-FO-115-D02 and IN-FO-115-CO1-SDB2-02).

1 Bonn D. Wild birds, poultry, and avian influenza. Lancet Infect Dis, 2006, 6: 262

2 Chen H, Smith G J, Zhang S Y, et al. H5N1 virus outbreak in migratory waterfowl. Nature, 2005, 436: 161-192

3 Davies P, Barry R D. Nucleic acid of influenza virus. Nature, 1966, 211: 384-387

4 Horimoto T, Kawaoka Y. Influenza: Lessons from past pandemic, warning from current incidents. Nat Rev Microbiol, 2005, 3: 591-600

5 Owoade A A, Ducatez M F, Muller C P. Seroprevalence of avian influenza virus, infectious bronchitis virus, reovirus, avian pneumovirus, infectious laryngotracheitis virus, and avian leukosis virus in Nigerian poultry. Avian Dis, 2006, 50: 222-227

6 Liu J H, Xiao H X, Lei F M, et al. Highly pathogenic H5N1 influenza virus infection in migratory birds. Science, 2005, 309: 1206

7 Guan Y. Genetic characterization of H5N1 viruses isolated from different regions of southern China. Hong Kong Med J, 2010, 16: 27-28

8 Kou Z, Li Y, Yin Z, et al. The survey of H5N1 flu virus in wild birds in 14 provinces of China from 2004 to 2007. PLoS ONE, 2009, 4: e6926

9 Lei F M, Tang S, Zhao D L, et al. Characterization of H5N1 influenza viruses isolated from migratory birds in Qinghai Province of China in 2006. Avian Dis, 2007, 51: 568-572

10 Li K S, Guan Y, Wang J, et al. Genesis of a highly pathogenic and potentially pandemic H5N1 influenza virus in eastern Asia. Nature, 2004, 430: 209-213

11 Smith G J, Vijaykrishna D, Ellis T M, et al. Characterization of avian influenza viruses A (H5N1) from wild birds, Hong Kong, 2004-2008. Emerg Infect Dis, 2009, 15: 402-407

12 Chen H, Smith G J, Li K S, et al. Establishment of multiple sublineages of H5N1 influenza virus in Asia: Implications for pandemic control. Proc Natl Acad Sci USA, 2006, 103: 2845-2850

13 Duan L, Campitelli L, Fan X H, et al. Characterization of lowhogenic H5 subtype influenza viruses from Eurasia: Implications for the origin of highly pathogenic H5N1 viruses. J Virol, 2007, 81: 7529-7539

14 Huang K, Bahl J, Fan X H, et al. Establishment of an H6N2 influenza virus lineage in domestic ducks in southern China. J Virol, 2010, 84: 6978-6986

15 Li Y, Shi J, Zhong G, et al. Continued evolution of H5N1 influenza viruses in wild birds, domestic poultry, and humans in China from 2004 to 2009. J Virol, 2010, 84: 8389-8397

16 Killian M L. Avian influenza virus sample types, collection, and handling. Methods Mol Biol, 2008, 436: 7-12

17 Nietfeld J C. Field necropsy techniques and proper specimen submission for investigation of emerging infectious diseases of food animals. Vet Clin North Am Food Anim Pract, 2010, 26: 1-13

18 Hoffmann E, Stech J, Guan Y, et al. Universal primer set for the full-length amplification of all influenza A viruses. Arch Virol, 2001, 146: 2275-2289

19 Munster V J, Baas C, Lexmond P, et al. Spatial, temporal, and species variation in prevalence of influenza A viruses in wild migratory birds. PLoS Pathog, 2007, 3: 630-638

20 Yang J L, Xia H, Zhao J R, et al. Molecular characterization of avian influenza virus (H7N8) isolated from poultry in Central China in the mid-1980s. Chin Sci Bull, 2010, 55: 1122-1127

21 Zhang H B, Chen Q J, Chen Z. Characterization of an H4N2 avian influenza virus isolated from domestic duck in Dongting Lake wetland in 2009. Virus Genes, 2012, 44: 24-31

22 Matrosovich M, Zhou N, Kawaoka Y, et al. The surface glycoproteins of $\mathrm{H} 5$ influenza viruses isolated from humans, chickens, and wild aquatic birds have distinguishable properties. J Virol, 1999, 73: 1146-1155

23 Suzuki H, Saito R, Masuda H, et al. Emergence of amantadineresistant influenza A viruses: Epidemiological study. J Infect Chemother, 2003, 9: 195-200

24 Seo S H, Hoffmann E, Webster R G. The NS1 gene of H5N1 influenza viruses circumvents the host anti-viral cytokine responses. Virus Res, 2004, 103: 107-113

Open Access This article is distributed under the terms of the Creative Commons Attribution License which permits any use, distribution, and reproduction in any medium, provided the original author(s) and source are credited. 